\title{
Research on Kruskal Crossover Genetic Algorithm for Multi- Objective Logistics Distribution Path Optimization
}

\author{
Yan Zhang ${ }^{1,2}$, Xing-yi Wu ${ }^{1}$ and Oh-kyoung Kwon ${ }^{2, a, \dagger}$ \\ ${ }^{1}$ Chongqing University of Posts and Telecommunications, Chongqing 400065, \\ China \\ ${ }^{2}$ Graduate School of Logistics, INHA University, Incheon 402751, Korea \\ ascm@inha.ac.kr \\ $\uparrow$ Corresponding - Author: Oh-kyoung Kwon
}

\begin{abstract}
To effectively optimize multi-objective logistics distribution path, the distance and distance related customer satisfaction factor are used as the objective function, a novel kruskal crossover genetic algorithm (KCGA) for multi-objective logistics distribution path optimization is proposed. To test the optimization results, the terminal distribution model and the virtual logistics system operating model are built. Experiment results show that, compared with basic genetic algorithm (GA), the run time of KCGA takes a slightly higher. But the average distribution distance and the best distribution distance are reduced by 6\%-8\%. Achieve the goal of multi-objective logistics distribution path optimization.
\end{abstract}

Keywords: logistics distribution; multi-objective; optimization; kruskal; crossover

\section{Introduction}

Along with the economic globalization and the development of national economy, logistics industry gets a great development. Because our logistics system is faultiness, since 2005, the ratio of logistics cost accounting for Chinese gross domestic product (GDP) has been around 18\% which is higher than Europe, America and other developed countries (which is around 10\%) [1]. In logistics system, a good distribution path can drive to get a better transport result, save resources and reduce cost efficiently. So far, the problem of logistics distribution path optimization has been a popular study point.

At present, the algorithms which are applied in logistics distribution path optimization mainly include ant colony algorithm (ACO) [2], genetic algorithm (GA) [3], particle swarm optimization (PSO) [4] and simulated annealing algorithm (SA) [5] and so on. Contrasting the algorithms mentioned above, based on biological evolution, GA starts searching from group and it can compare multiple individuals at the same time. In other words, GA has potential parallelism and high overall. According to these advantages, GA is a good choice for logistics distribution path optimization.

GA was first presented by J.Holland in 1975 [6]. Except the advantages above, "earlymaturing" is the weakness of GA [7-8] and it can lead logistics distribution path not achieving optimal. To cure above problems, Ombuki [9] present a hybrid Tabu-search/GA algorithm. The number of vehicles was optimized by GA and the distance was optimized by Tabu-search algorithm. The hybrid algorithm can obtain a better result than anyone of Tabu-search and GA. Tseng [10] introduced the probability theory into GA. The probability was regard as a parameter which was used to adjust crossover and mutation model. In the meantime, a two-way method was applied in global optimization search.

Taking aim at the problems of long distribution path and time by using GA to optimize distribution path, this paper introduces kruskal algorithm into GA. The delivery distance 
and customer satisfaction which are regard as objective function are present into this new method. According to vehicle routing problem (VRP) model, order terminal distribution model which is used to verify the proposed method is structured. So, this method can be accomplished in the distribution model.

\section{Multi-Objective Logistics Distribution Path Optimization}

\subsection{Objective Function}

Total distribution distance objective function: in logistics system, delivery paths are the routes of vehicles. The length decides the distribution quality and cost. So the total distribution distance is the prior objective function for optimizing.

Customer satisfaction objective function: on the premise of the minimum total distribution distance, customer satisfaction is a very important indicator. It can influence the clients directly and then the competition ability and economic benefit of enterprises [11].

In express logistics industries, Customer satisfaction is mainly decided by the arrival time of goods. Customer satisfaction objective function $F_{i}\left(t_{i}\right)$ is shown in (2).

$$
\begin{gathered}
f_{i}\left(t_{i}\right)= \begin{cases}1 \quad & \begin{array}{ll}
\frac{L L_{i}-t_{i}}{L L_{i}-L_{i}} ; & L_{i}<t_{i} \leqslant L L_{i}
\end{array} \\
0 \quad ; \quad t_{i}>L L_{i}\end{cases} \\
F_{i}\left(t_{i}\right)=f_{i}\left(t_{i}\right) * X_{i}
\end{gathered}
$$

Where $t_{i}$ is the arrival time of goods, the satisfying time is range in $\left[0, L_{i}\right] . L L_{i}$ presents the maximum time. As shown in Figure 1 , in $\left[L_{i}, L L_{i}\right], F_{i}\left(t_{i}\right)$ reduces to 0 gradually. $X_{i}$ is the number of orders.

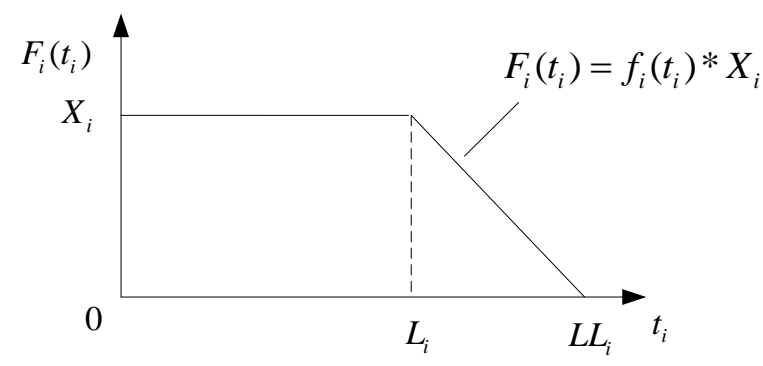

Figure 1. Customer Satisfaction Change Along with the Goods Arrival Time

Total objective function: total objective function is composed of negative $F_{i}\left(t_{i}\right)$ and total distribution distance objective function. Then minimum value is obtained by using GA. Total objective function is shown in (3).

$$
F_{i}\left(t_{i}\right)_{0}=\left(-F_{i}\left(t_{i}\right)\right)+F_{i}\left(t_{i}\right)_{1}
$$

Where $F_{i}\left(t_{i}\right)_{0}$ represents the total objective function, $F_{i}\left(t_{i}\right)$ represents the customer satisfaction objective function, $F_{i}\left(t_{i}\right)_{1}$ represents the total distribution distance objective function. 


\subsection{Kruskal Algorithm}

Kruskal algorithm is a kind of greedy algorithm. It builds a minimum cost spanning tree by adding one edge to the cost spanning tree at every turn. Namely, kruskal algorithm can achieve global optimization according to local optimal choice [12]. Suppose that $\mathrm{WN}=(\mathrm{V},\{\mathrm{E}\})$ is a connected net which includes $\mathrm{n}$ peaks. Then, the process of building minimum spanning tree (MST) using kruskal algorithm can be described as follows.

(1) Build a sub graph which only includes $n$ peaks and its frontier sets are empty;

(2) Find one edge with the smallest weight from the frontier set $E$ of the sub graph. If the two peaks of the edge are belonging to different trees, add the edge to the sub graph;

(3) If the two peaks are part of one tree, give up this edge. Then find the next edge with smallest weight and repeat (2) and (3) until there are n-1 edges in sub graph.

The pseudo code of kruskal algorithm is as follows.

Begin

$\mathrm{T} . \mathrm{V}=\mathrm{n} ; \mathrm{T} . \mathrm{E}=\{\}$;

For ( $\mathrm{n}-1$ edges in the sub graph)

Find the shortest edge (u,v) from WN.E;

If ( $u, v$ local in two different unicom components of $T$ )

Add ( $u, v)$ to T.E;

Else

Select the second shortest edge;

End if;

End for

End

\subsection{Terminal Distribution Model}

In this paper, combining with customer satisfaction objective function and VPR model, the terminal distribution model, which includes a distribution center and multiple distribution terminals, is established. The VRP model can be described as this: in this model, some trucks which have a fixed load are used to deliver cargoes from logistics center to different destinations, and every destination has not only a fixed position but also different demands for goods [13]. In order to make the objective function minimum or maximum and to meet the following constraint conditions, the path ought to be conFigured reasonably. The constraint conditions are described as below.

(1) In some distribution path, the total requirements of destinations should be less than lorries` total loads;

(2) The length of some distribution path should be shorter than the maximum mileages of vehicles;

(3) Every destination's requirements should be met and the goods should be delivered by a truck only.

(4) During the distribution, every destination should be reached once. After that, cars can go back to distribution center.

\subsection{Kruskal Crossover Genetic Algorithm (KCGA)}

Because basic GA is easy to converge, when distribution path are optimized by this method, a problem that the calculated path are always too long appears. Kruskal is a greedy algorithm which establishes the final minimum spanning tree by adding an edge into the spanning tree every time, and it has the characteristic that global optimization can be achieved through local optimization. Thus, in this paper, the algorithm is introduced into the crossover operator of basic GA to increase the diversity of population. Further, it 
is used to assure that the algorithm can find out the optimum of individuals during the cross operation and to solve the premature convergence problem in basic GA.

The flow chart of KCGA is shown as Figure 2.

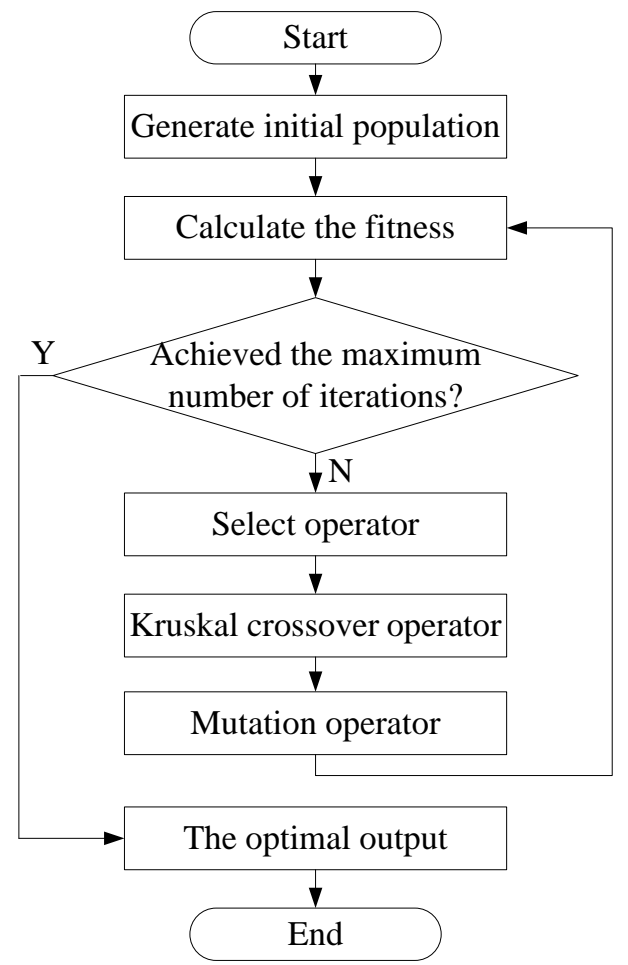

Figure 2. The Flow Chart of KCGA

KCGA is designed as below.

(1) Two parent individuals FA and FB are obtained by selection steps and two gene fragments FA1 and FB1 with random length are selected from these two individuals as cross genes;

(2) Use kruskal algorithm to search FA1 and FB1, and add the shorter and shortest sides of FA1 and FB1 into FA2 and FB2 respectively, then remove the repetitive parts of FA2 and FB2 to get the new FA2 and FB2;

(3) Remove the FA's repetitive parts which is contained in FB2. So does FB by taking FA2 into consideration;

(4) Exchange FA2 and FB2, then combine FA and FB2 to get the new offspring SA. Similarly, SB is obtained by combining FB and FA2

(5) Introduce SA and SB into the rest of basic GA to get the optimal solution by iteration.

Where step (2) is achieved by the following pseudo codes.

Begin

Input FA1; FB1;

If (FA1 meets the conditions of minimum spanning tree)

FA2 $=($ FA1 (shortest edge), FA1 (shorter edge)...);

//Establish the minimum spanning tree

$\mathrm{FB} 2=(\mathrm{FB} 1$ (shortest edge), FB1 (shorter edge)...);

Else

Give up the edges which cannot meet the conditions and search for next edge;

End if 
Del (FA2); Del (FB2); //Remove repetitive genes

Return FA2; FB2;

End

For example, $\mathrm{FA}=32416758, \mathrm{FB}=32148765(1,2,3 \ldots$ are the gene points). Take out the gene fragment FB1=1487 of parental gene FB. Rand every edges of FB1 by ascending counts, i.e., $(1,4),(4,7),(1,7),(4,8),(8,7),(1,8)$. As a result, FB2 can be getting by step 2 in the following way.

a. $\mathrm{FB} 2=(144748) ; / /$ Establish the minimum spanning tree. Based on kruskal to examine every edge of FB1, conditions of $(1,4)$ and $(4,7)$ are introduced first. When condition $(1,7)$ is introduced, the rules of minimum spanning tree cannot be met. Therefore, instead of giving up edge $(1,7)$, we use the algorithm to search for next one $(4$, $8)$. As a result, the minimum spanning tree is established by adding the new edge $(4,8)$.

b. Remove the repetitive genes, we get FB2 $=1478$.

The solution of FA2 is similar to that of FB2.

Where step (4) is achieved by the following pseudo codes.

Begin

Input FA; FB; FA2; FB2;

Wipe out the same gene between FA and FB2 and also between FB and FA2;

$\mathrm{SA}=(\mathrm{FA}, \mathrm{FB} 2) ; \mathrm{SB}=(\mathrm{FB}, \mathrm{FA} 2)$;

Return SA; SB;

End

Follow the above example, the result can be obtained as follows. FB2 $=1478$, $\mathrm{FA}=32416758$. Thus, Step (4) can be realized as follows.

a. Wipe out the same gene between FA and FB2. FA=3265;

b. $\mathrm{SA}=(\mathrm{FA}, \mathrm{FB} 2)=32651478$.

The method to solve SB is similar with that of SA.

However, after add kruskal algorithm to the basic GA crossover operator, increased the overall complexity of the algorithm, and the corresponding algorithm run time consuming also increased. Therefore, this paper makes a further optimized to KCGA by the following methods, which will effectively reduce the algorithm running time consuming.

(1) Control algorithm computation. Initial kruskal crossover extracts a random length of the parent application kruskal algorithm. It is likely to take the long gene segments of the parent generation (e.g. more than $60 \%$ of the length). Thus, by the method of controlling the length of the gene, which take no more than $20 \%$ of the total length of genes when the number of terminal city point is smaller (the number of terminals city point below 50), take no more than $10 \%$ of the total length of genes when the number of terminal city point is greater (the number of terminals city point above 50). It is in order to effectively control the computational of algorithm.

(2) Add initial population elitist strategy and offspring elite strategy.

a. Initial population elite strategy is initialize the initial population for many times, take out the $\mathrm{n}$ individual in the top initialize objective function values every time as the final part of the initialization population, constitute the final initial population the after repeated many times. For example, if the initial population is set to 50 units, it cans initialization by 10 times, reserve the 5 units in the top objective function value, and constitute the final initialization population after repeat 10 times.

b. The strategy of offspring elite is after selection, crossover and mutation operation, retain the best unit. This unit does not participate in crossover and mutation operation of the next generation, which only participates in the choice computing of the next generation. It is in order to accelerate the algorithm convergence rate.

Therefore, KCGA pseudo code as follows.

Begin 


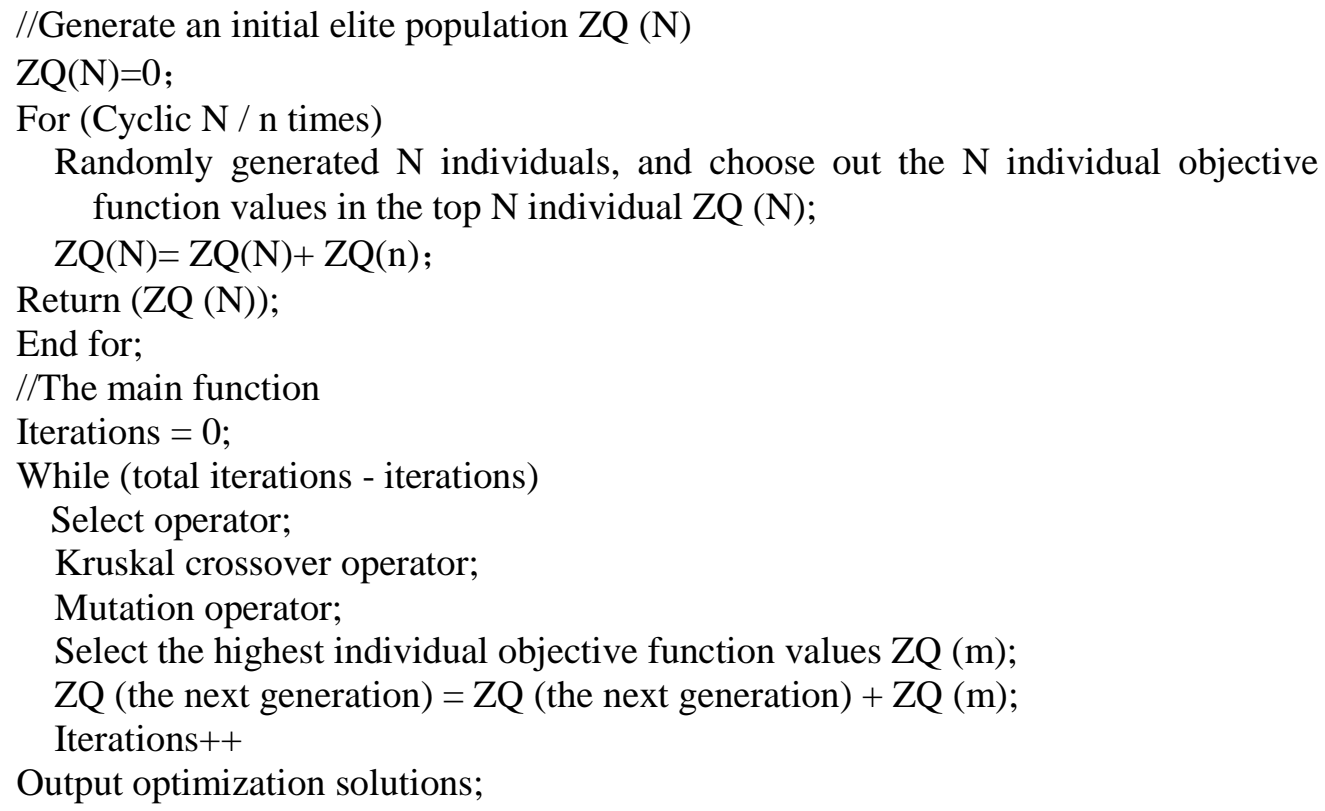
End;

Select the tied for choice method to solve multi-objective optimization, which can provide multiple parallel selection methods.

(1) The population is divided into three equal sub-populations according to the number of objective function.

(2) Assigned an objective function to each group.

(3) New population will complete crossover and mutation operations after each subobjective function complete selection operation independently in their corresponding subgroups .

(4) Iterative to get optimal path.

\section{Experimental Results and Analysis}

\subsection{Comparative Experiment of Using Basic GA and KCGA to Solve the Order Terminal Distribution Model, Which Makes the Distribution Distance as the Objective Function}

Build the order terminal distribution model in one distribution center and different terminal points. Make the distribution distance as the objective function, and use the basic GA and KCGA to solve this model ten times. At last, the result of the experiment will be analyzed.

The operation parameters of the two algorithms set as follows.

The two algorithms are added with initial population elite strategy and offspring elite retention policy.

The location of test city point and the number of orders are generated by random.

The population size is 50 .

The evolution algebra is 100 .

The crossover probability is 0.8 .

The mutation probability is 0.1 .

The distribution vehicle load restraint is $250 \mathrm{~kg}$.

The distribution vehicle distance constraints are $80 \mathrm{~km}$.

The experiments were conducted on an Intel 2.17GHZ Windows 7 platform with $2 \mathrm{~GB}$ RAM, and algorithm is implemented in MATLAB. The experimental results are shown in Table 1. 
Table 1. The Two Methods' Experimental Results

\begin{tabular}{|c|c|c|c|c|c|}
\hline $\begin{array}{l}\text { Number } \\
\text { of city }\end{array}$ & $\begin{array}{l}\text { Algorit } \\
\mathrm{hm}\end{array}$ & $\begin{array}{l}\text { Distribution distance } \\
\text { (Ten times, unit: } \mathrm{km} \text { ) }\end{array}$ & $\begin{array}{c}\text { Average } \\
\text { time } \\
\text { (s) }\end{array}$ & $\begin{array}{c}\text { Average } \\
\text { distance } \\
(\mathrm{km})\end{array}$ & $\begin{array}{c}\text { Optimal } \\
\text { distance } \\
(\mathrm{km})\end{array}$ \\
\hline \multirow{2}{*}{10} & GA & $71,71,68,70,68,68,68,69,69,70$ & 13.79 & 69.2 & 68 \\
\hline & KCGA & $68,68,68,68,68,68,68,68,68,68$ & 16.56 & 68 & 68 \\
\hline \multirow{2}{*}{15} & GA & $124,118,114,117,126,122,120,120,117,119$ & 18.61 & 119.7 & 114 \\
\hline & KCGA & $113,113,113,113,114,113,113,114,113,114$ & 20.96 & 113.3 & 113 \\
\hline \multirow{2}{*}{20} & GA & $139,135,134,135,141,141,133,139,149,140$ & 25.28 & 138.6 & 133 \\
\hline & KCGA & $129,125,139,130,128,131,135,125,130,129$ & 43.84 & 130.1 & 125 \\
\hline \multirow{2}{*}{25} & GA & $155,151,160,156,151,159,166,162,156,149$ & 37.02 & 156.5 & 149 \\
\hline & KCGA & $144,141,149,139,139,139,147,144,138,139$ & 60.50 & 141.9 & 138 \\
\hline \multirow{2}{*}{30} & GA & $200,209,204,198,212,189,199,195,193,205$ & 45.11 & 200.4 & 193 \\
\hline & KCGA & $178,186,178,190,182,188,176,180,186,188$ & 72.72 & 183.2 & 176 \\
\hline \multirow{2}{*}{40} & GA & $261,264,248,251,264,259,269,267,260,252$ & 50.81 & 259.5 & 248 \\
\hline & KCGA & $246,240,229,252,239,235,240,245,229,231$ & 91.39 & 238.6 & 229 \\
\hline \multirow{2}{*}{50} & GA & $357,361,369,345,345,340,343,351,353,355$ & 69.06 & 351.9 & 340 \\
\hline & KCGA & $344,319,335,337,325,338,335,310,320,325$ & 126.48 & 328.8 & 310 \\
\hline \multirow{2}{*}{75} & GA & $558,543,566,563,555,548,558,570,550,539$ & 120.03 & 555 & 539 \\
\hline & KCGA & $523,540,512,539,497,521,525,503,509,538$ & 135.65 & 520.7 & 497 \\
\hline \multirow{2}{*}{100} & GA & $701,717,690,682,737,734,711,702,695,683$ & 146.89 & 705.2 & 682 \\
\hline & KCGA & $676,643,658,606,669,648,662,605,647,663$ & 196.70 & 647.7 & 605 \\
\hline
\end{tabular}

Table 1 show that the two algorithms were run 10 times, compared with basic GA, the run time of KCGA takes a slightly higher (higher than the range of 10\%-80\%). However, when comparing the results of the optimal distance and the average distance in this ten times, KCGA is not inferior to GA. For example, with 15 terminal city points, the average distance that solved by KCGA was reduced by $5.35 \%$, and the optimal distance was reduced by $0.88 \%$. When there are 30 terminal city points, the average distance that solved by KCGA was reduced by $8.58 \%$, and the optimal distance was reduced by $8.81 \%$. When there are 50 terminal city points, the average distance that solved by KCGA was reduced by $6.56 \%$, the optimal distance was reduced by $8.82 \%$. When there are 75 terminal city points, the average distance that solved by KCGA was reduced by $6.18 \%$, the optimal distance was reduced by $7.79 \%$.

\subsection{Experiment of Using Basic GA and KCGA to Solve the Order Terminal Distribution Model, which Makes the Distribution Distance and Customer Satisfaction as the Objective Function}

Order terminal distribution model is built by a distribution center and fifteen terminal cities. Where, distribution center is located in $[8,8]$, the quality of each accessory is randomly generated from 0 to 10 kilogram, the rated load and speed of vehicles are $400 \mathrm{~kg}$ and $30 \mathrm{~km} / \mathrm{h}(0.5 \mathrm{~km} / \mathrm{min})$, and the server time of distribution vehicle is 15 minutes in each distribution point, and the rest of parameters are consistent with the previous section experiment.

Table 2. Terminal Point Coordinates the Number of Orders and Time Constraints

\begin{tabular}{|c|c|c|c|c|c|}
\hline \multirow{2}{*}{$\begin{array}{l}\text { Terminal } \\
\text { point }\end{array}$} & \multirow{2}{*}{$\begin{array}{c}\text { Number of orders } \\
\qquad X_{i} \text { (piece) }\end{array}$} & \multicolumn{2}{|c|}{ Coordinates } & \multirow{2}{*}{$\begin{array}{c}\text { Time constraints } \\
{\left[L_{i}, L L_{i}\right]} \\
(\mathrm{min})\end{array}$} & \multirow{2}{*}{$\begin{array}{l}\text { Service time } \\
\quad(\min )\end{array}$} \\
\hline & & $X$ & $\mathrm{Y}$ & & \\
\hline 1 & 34 & 3 & 2 & {$[60,80]$} & 15 \\
\hline 2 & 25 & 1 & 5 & {$[60,80]$} & 15 \\
\hline
\end{tabular}




\begin{tabular}{|l|c|c|c|c|c|}
\hline 3 & 46 & 5 & 4 & {$[60,80]$} & 15 \\
\hline 4 & 15 & 4 & 7 & {$[60,80]$} & 15 \\
\hline 5 & 5 & 0 & 8 & {$[60,80]$} & 15 \\
\hline 6 & 55 & 3 & 11 & {$[60,80]$} & 15 \\
\hline 7 & 65 & 7 & 9 & {$[60,80]$} & 15 \\
\hline 8 & 43 & 9 & 6 & {$[60,80]$} & 15 \\
\hline 9 & 34 & 10 & 2 & {$[60,80]$} & 15 \\
\hline 10 & 10 & 14 & 0 & {$[60,80]$} & 15 \\
\hline 11 & 8 & 17 & 3 & {$[60,80]$} & 15 \\
\hline 12 & 43 & 14 & 6 & {$[60,80]$} & 15 \\
\hline 13 & 32 & 12 & 9 & {$[60,80]$} & 15 \\
\hline 14 & 44 & 10 & 12 & {$[60,80]$} & 15 \\
\hline 15 & 14 & 7 & 14 & {$[60,80]$} & 15 \\
\hline
\end{tabular}

Experiments are performed with KCGA is applied into the model. The experiments were conducted on an Intel 2.17GHZ Windows 7 platform with 2GB RAM, and algorithm is implemented in MATLAB. Results are showed as follows.

(1) The shortest distribution distance

a. Distribution path: 0-9-10-11-12-0; 0-8-13-14-15-0; 0-1-3-0; 0-7-0; 0-6-5-2-4-0;

b. Number of vehicles: 5

c. Distribution distance: 82

d. Customer satisfaction (the shortest distribution distance): 132.92

(2) The highest customer satisfaction

a. Distribution path: 0-13-14-15-0; 0-7-0; 0-6-5-2-0;0-3-1-4-0;0-8-9-10-0;0-12-110 ;

b. Number of vehicles: 6

c. Distribution distance (the highest customer satisfaction): 97

d. Customer satisfaction: 155.80

(3) The comprehensive objective function optimal

a. Distribution path: 0-3-1-4-0; 0-8-12-13-0; 0-6-5-2-0; 0-7-15-14-0; 0-9-10-11-0;

b. Number of vehicles: 5

c. Distribution distance: 91

d. Customer satisfaction: 155.49

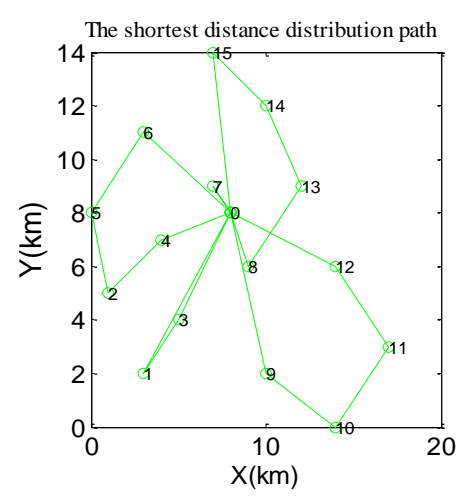

(a)

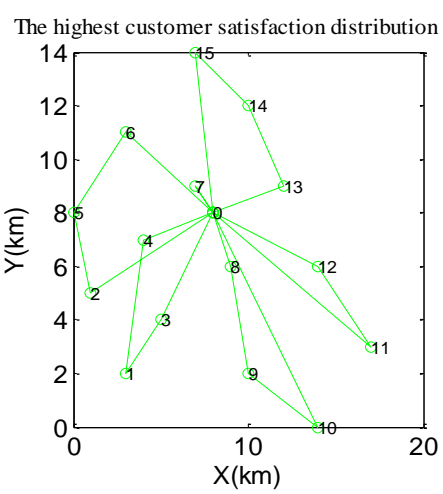

(b)

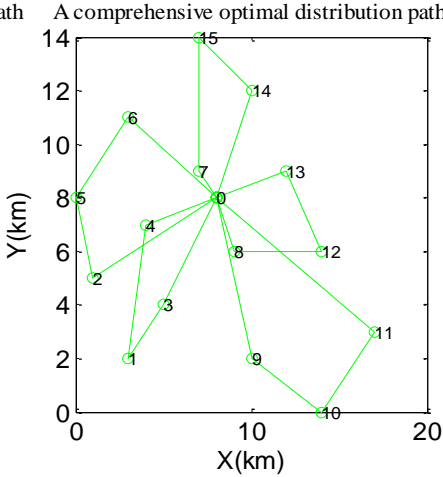

(c)

Figure 3. The Distribution Path Chart of KCGA Solve the Model 


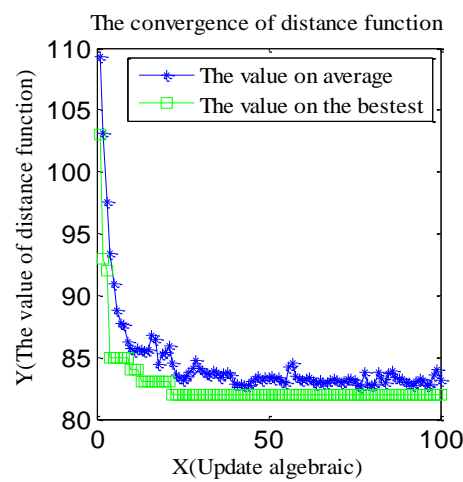

(a)

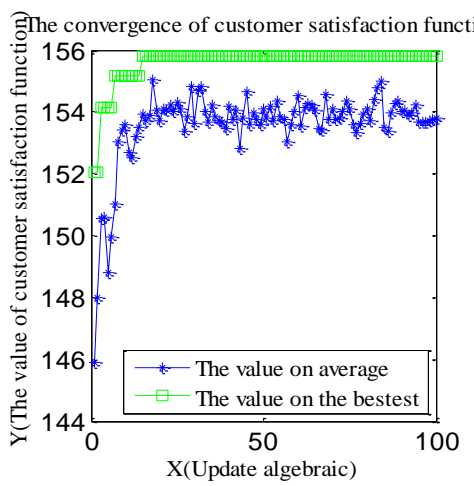

(b)

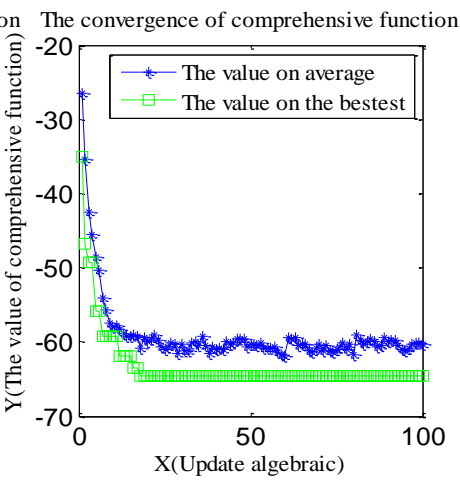

(c)

\section{Figure 4. The Objective Function Value Convergence Effect Chart of KCGA Solve the Model}

Combining Figure 3, 4 and the experiment results, we could found that the distribution distance and customer satisfaction are lower than the single optimal value when the comprehensive objective function optimal. However, another objective function value is lower than the result of comprehensive objective function optimal when the single objective function is optimal. Therefore, distribution path will be closer to the real situation when the customer satisfaction objective function is added, and the distribution plan of three different advantages can be provided.

\subsection{Distribution Path Optimization Experiment Under Virtual Logistics System}

Logistics space model is composed by a logistics center of tier one city, a distribution center of secondary city, and a logistics distribution point of terminal city. In addition, order generate model, storage model, transport and terminal distribution model are essential for the simple model of virtual logistics system. This model is implemented in MATLAB/GUI.

The operating principles are defined as follows: orders are distributed from a starting point, and sending these orders to the distribution center, which is belonging to the city of order creation. Then, these orders will be delivered from distribution center to the previous level that is logistics center. After all kinds of orders have been processed and classified, sending each order to the corresponding logistics center of destination city. Next, delivering orders from logistics center to the next level that is distribution center. At last, each order will arrive to their destination thorough distribution center. Where, order terminal distribution module is belongs to the simple model of virtual logistics system, and the successful delivery of orders from secondary city to the terminal city is the main function for the module.

Configure the system parameters and generate the city architecture. Architecture generated Figure of the system main interface as shown in Figure 5. 


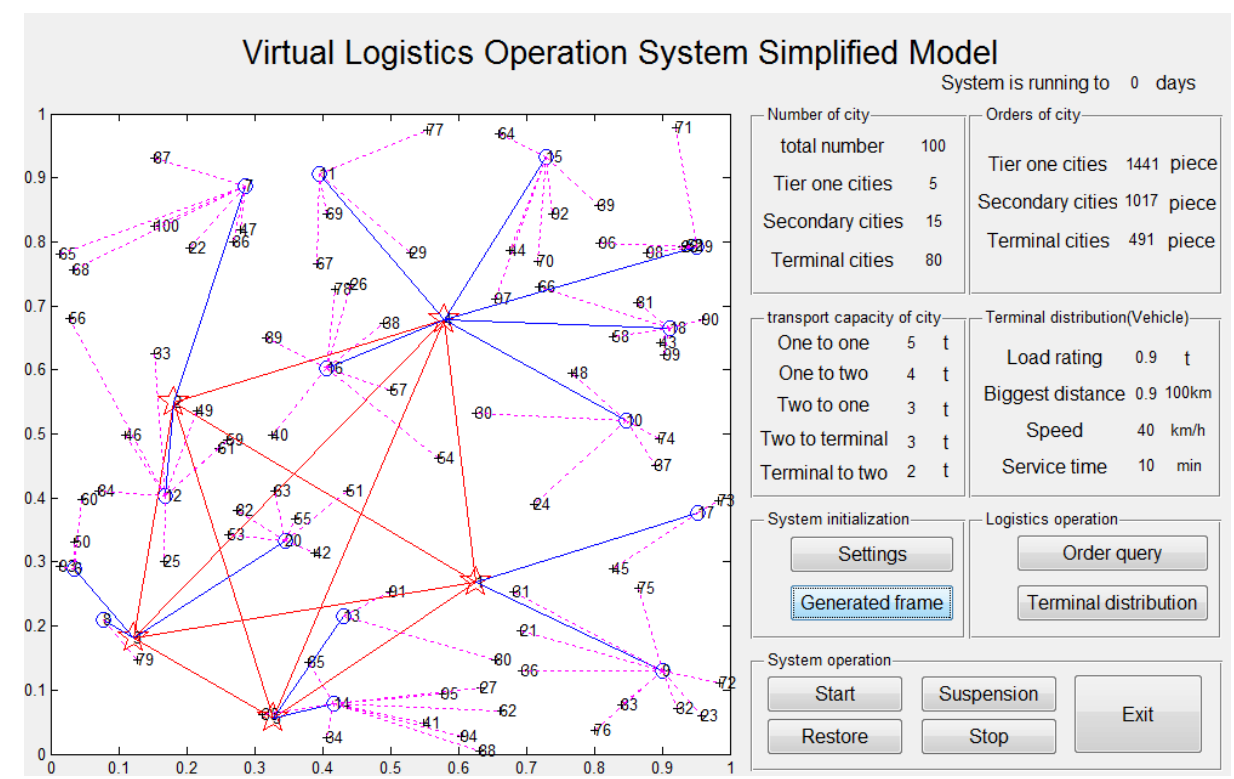

Figure 5. The Main Interface Architecture Chart of System

As the Figure 5 shows, the dotted line in the Figure is terminal city points to secondary cities, which represents a running route between these two points. The solid line is secondary cities to tier one city and between tier one cities, it also represents a running route. Run the system and enter the terminal distribution module, random selection on January 2, 9th secondary city for testing. The test results are shown in Figure 6.

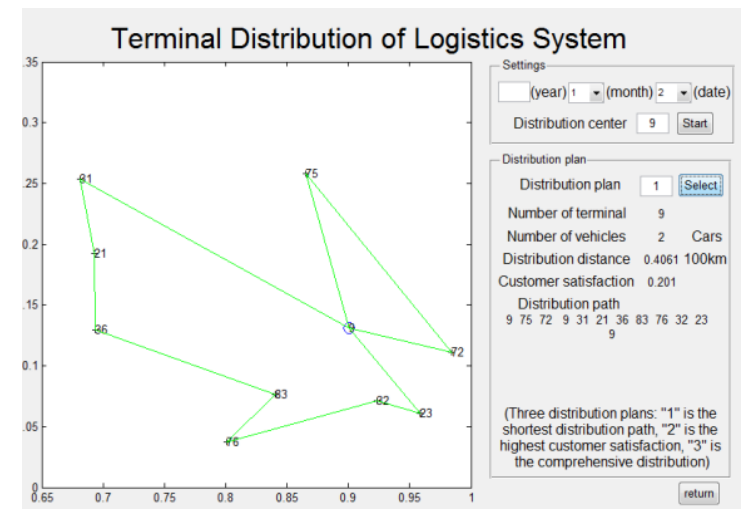

(a)

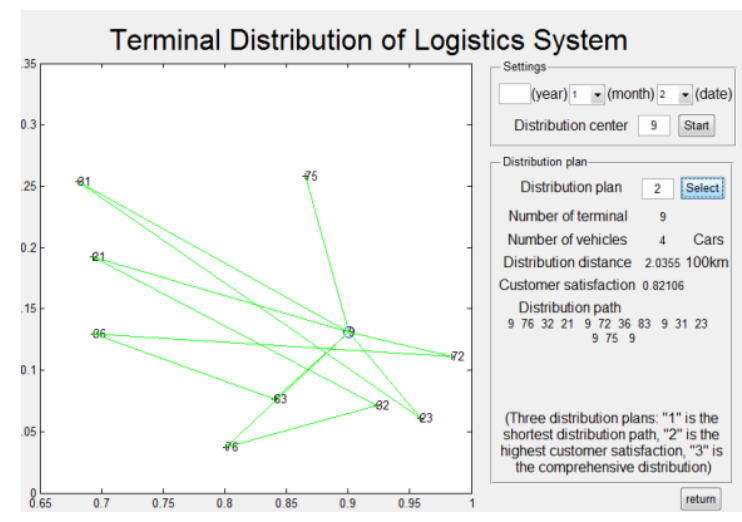

(b) 


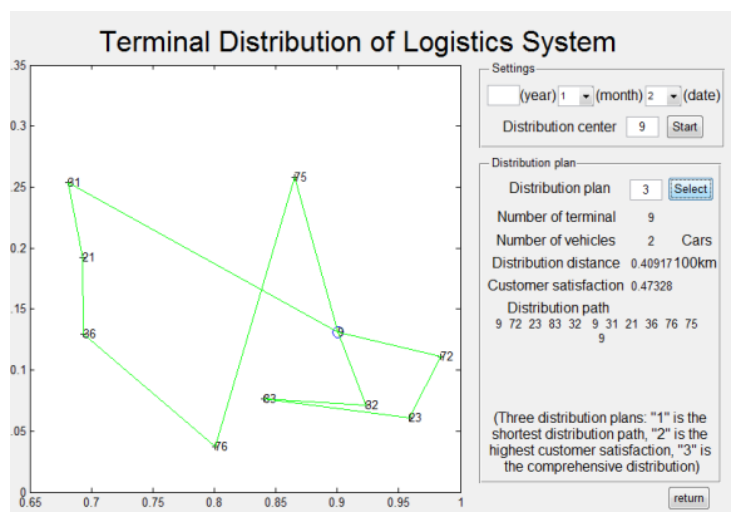

(c)

\section{Figure6. The Distribution Path Chart of System Terminal Distribution Module}

As the Figure 6 shows, the distribution module can provide three kinds of distribution plan as follow. The first is the shortest distribution path, second is the highest customer satisfaction, and the last is comprehensive distribution. In Figure 6(a), choose the first distribution plan, the distribution distance is $40.61 \mathrm{~km}$ and the customer satisfaction is 0.2010. In Figure 6(b), choose the second distribution plan, the distribution distance is $203.55 \mathrm{~km}$ and the customer satisfaction is 0.8211 . In Figure 6(c), choose the last distribution plan, the distribution distance is $40.92 \mathrm{~km}$ and the customer satisfaction is 0.4733 . The experimental results are same as the experimental 3.2 shows, which the algorithm is proved effective.

\section{Conclusion}

Taking aim at the problems of long distribution routes and time by using basic GA to optimize multi-objective logistics distribution path, this paper use the distance and distance related customer satisfaction factor as the objective function, and introduces kruskal algorithm into basic GA to achieve the optimizing multi-objective logistics distribution path. At last, to test the optimization results, the terminal distribution model and the virtual logistics system operating model are built. Experiment results show that, compared with basic GA, the run time of KCGA takes a slightly higher. But the average distribution distance and the best distribution distance are reduced by $6 \%-8 \%$. Achieve the goal of multi-objective logistics distribution path optimization.

\section{Acknowledgments}

This work is supported by Science Foundation Project of CQ (CSTC2013yykfC60005, cstc2014jcyjA60004).

\section{References}

[1] Zhao D. M., "The Quantitative and Marginal Analysis of Reducing the Compassion between the Logistics Costs and GDP," Logistics Engineering and Management, vol. 34, (2012), pp. 3-5.

[2] Li Z., Chen X. H. and Wang H., "Buckling Optimization of Composite Laminate Using Modified Ant Colony Optimization,” J. Shanghai Jiaotong Univ. (Sci.), vol.46, (2012), pp.768-773.

[3] Guo Y., Lin Y. and Zhang S., "Interactive genetic algorithms based on frequent-pattern mining," Proceedings - 2010 6th International Conference on Natural Computation (ICNC), (2010), pp. 23812385.

[4] Wang T. Z. and Fan G. L., "The research of building fuzzy C-means clustering model based on particle swarm optimization," Telkomnika - Indonesian Journal of Electrical Engineering, vol. 11, (2013), pp.7589-7598. 
[5] Li Y., Jiang J., Zhang Min X., “Automatic Word-Length Determination Tool Based on Simulated Annealing Algorithm," J. Shanghai Jiaotong Univ. (Sci.), vol.47, (2013), pp.76-80.

[6] Holland J., "Genetic Algorithm Theory and Application," Translation of Li Min-qiang. Beijing science and Technology Press, (2003).

[7] Kosinski. W., Kotowski S. and Michalewicz Z., "On convergence and optimality of genetic algorithms," 2010 IEEE Congress on Evolutionary Computation (CEC), (2010), pp.1-6.

[8] Feng L., Chunyan Z. and K. C. Chang, "Convergence rate analysis of allied genetic algorithm," 2010 49th IEEE Conference on Decision and Control (CDC), (2010), pp.786-791.

[9] B. Ombuki, M. Nakamura and M. Osamu, "A Hybrid Search Based on Genetic Algorithms and Tabu Search for Vehicle Routing," In the 6th International Conference on Artificial Intelligence and Soft Computing .Banff [C].Canada: ABC, (2002), pp.176-181.

[10] Tseng L. T., "Genetic algorithm using the bimodal operation to prevent prematurity and reduce computational time," Applied Mechanics and Materials, (2012), pp.1987-1991.

[11] Shi L., "Supply chain partnership research based on customer satisfaction degree," Proceedings of the 2012 2nd International Conference on Business Computing and Global Informatization, (2012), pp. 203205.

[12] Osipov V., (Universität Karlsruhe (TH), Germany); Sanders P., Singler J., "The Filter-Kruskal minimum spanning tree algorithm," 2009 Proceedings of the 11th Workshop on Algorithm Engineering and Experiments, (2009), pp. 52-61.

[13] Wu Q., "VRP optimization of intensive distribution in enterprise sales logistics," 2010 International Conference on Management and Service Science, (2010), pp.1-3.

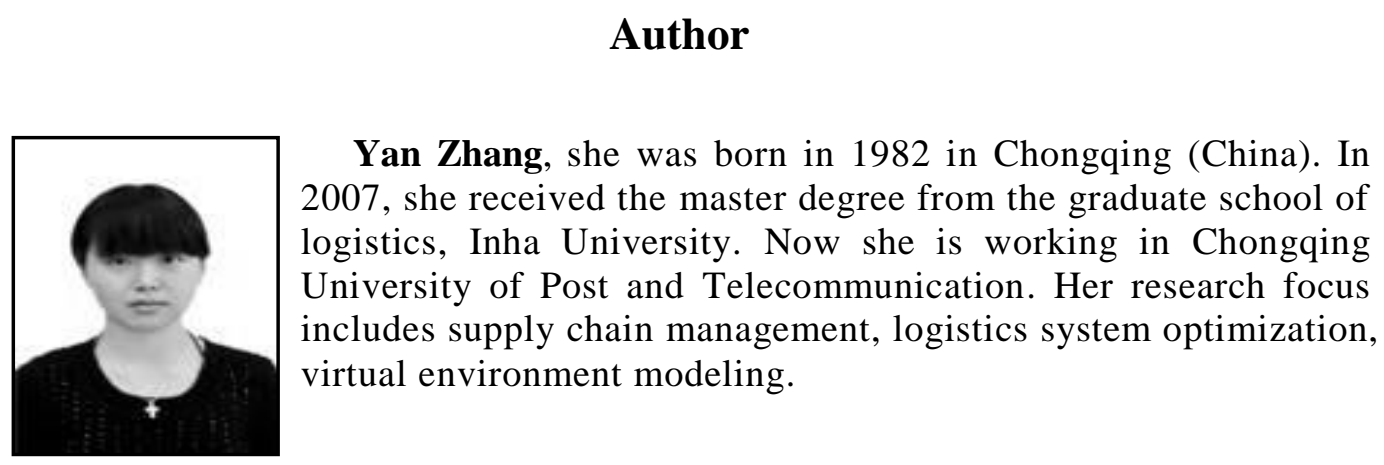

